\title{
Diagnostic accuracy of high-risk HPV genotyping in women with high-grade cervical lesions: evidence for improving the cervical cancer screening strategy in China
}

\author{
Huihui Xu ${ }^{1}$, Aifen Lin ${ }^{2}$, Xiujuan Shao ${ }^{3}$, Weiwu Shi ${ }^{1}$, Yang Zhang ${ }^{1}$ and Weihua Yan ${ }^{1}$ \\ ${ }^{1}$ Medical Research Center, Taizhou Hospital of Zhejiang Province, Wenzhou Medical University, Linhai, Zhejiang, China \\ ${ }^{2}$ Human Tissue Bank, Taizhou Hospital of Zhejiang Province, Wenzhou Medical University, Linhai, Zhejiang, China \\ ${ }^{3}$ Department of Gynecology, Taizhou Hospital of Zhejiang Province, Wenzhou Medical University, Linhai, Zhejiang, China \\ Correspondence to: Weihua Yan, email: yanwhcom@yahoo.com \\ Keywords: high-risk human papillomavirus, genotyping, diagnosis, high-grade cervical lesion, cervical cancer screening \\ Received: June 13,2016 Accepted: September 05, $2016 \quad$ Published: September 10, 2016
}

\section{ABSTRACT}

Currently, clinical data for primary HPV screening alone are lacking in China. Here, we evaluate cervical cancer screening with primary HPV genotyping, as well as possible future screening strategy. Overall, high-risk HPV (hrHPV) prevalence was 18.2\% among hospital-based population in Taizhou area. For cervical intraepithelial neoplasia 2 or worse (CIN2+), the sensitivity of primary hrHPV genotyping strategy and current cervical cancer screening strategy were $93.5 \%$, and $71.1 \%$, respectively; whereas the specificity was $17.5 \%$, and $62.4 \%$, respectively. Current cervical screening strategy had slightly higher positive predictive values (28.4\%) for CIN2+ than hrHPV genotyping strategy $(21.9 \%)$, whereas primary hrHPV genotyping strategy demonstrated higher negative predictive values $(94.7 \%)$ than current cervical screening strategy (91.1\%). Compared to HPV35/39/45/51/56/59/66/68 genotypes, the odds ratios (OR) for CIN2+ in HPV16/18/31/33/52/58 infection women were 3.2 (95\% confidence interval [CI] 2.3-4.1). Primary hrHPV genotyping strategy provides a better predictive value than HPV16/18 genotyping alone in guiding the clinical management of the current cervical cancer screening. HPV testing without adjunctive cytology may be sufficiently sensitive for primary cervical cancer screening.

\section{INTRODUCTION}

Worldwide, cervical cancer is the second most common female malignancy. Approximately 500,000 new cases of cervical cancer are diagnosed and 275,000 deaths from cervical cancer occur annually. Persistent infection of high-risk human papillomavirus (hrHPV) is necessary for the development of high-grade intraepithelial neoplasia (CIN2/3) and cervical cancer [1]. More than 100 types of HPV can infect the anogenital epithelium, of which at least 14 types are classified as high-risk because of their strong carcinogenic potentials; sexually transmitted HPV may lead to cervical carcinogenesis [2]. HPV16 and HPV18 are the two most carcinogenic genotypes, accounting for $55-60 \%$ and $10-15 \%$ of cervical cancers, respectively. Additionally, HPV31, 33, 35, 39, 45, 51, 52, 56, 58, 59, 66 , and 68 are also closely associated with cervical cancer. Significantly, epidemiologic studies have shown that nearly $100 \%$ of patients with cervical cancer test positive for HPV.

Public health screening programmes have successfully decreased cervical cancer incidence and mortality, including cervical cancer screenings and HPV vaccinations. Current guidelines for cervical cancer screening which cosponsored by the American Cancer Society (ACS), the American Society for Colposcopy and Cervical Pathology (ASCCP), and the American Society for Clinical Pathology (ASCP) in 2012, were recommendations address age-appropriate screening strategies, including the use of cytology and hrHPV testing (co-testing) [3, 4]. Recently, American Food and Drug Administration (FDA) approval hrHPV testing as an option for primary screening, which use of HPV16/18 genotyping along with a cocktail test of 12 other hrHPV genotypes [5]. However, genotyping solely for HPV16/18 would miss the majority of patients with low-grade 
squamous intraepithelial lesion (LSIL) who progress to high-grade cervical lesions [6]. In addition, because of the differences between the European/ United States populations in terms of the screening frequency, HPV genotypic distribution, and HPV vaccination rates, the data collected from these countries may not represent the situation in China.

With the aim to establish a foundation for primary HPV screening in a certain area, and to support the local vaccination program in Taizhou region. This populationbased, prospective observational study was designed to analyze the distribution of individual hrHPV genotypes across the complete spectrum of cervical disease; we have performed the primary HPV screening in detecting precancerous high-grade cervical lesions and cervical cancer.

\section{MATERIALS AND METHODS}

\section{Study population}

The Taizhou Area HPV study is a population-based, prospective observational study. We used HPV genotyping for primary cervical screening strategy, women with screen results of hrHPV positive referred directly to colposcopy biopsy. Moreover, the current cervical screening strategy with cytology and hrHPV testing (co-testing), the management of screen results stratified follow by: 1) atypical squamous cells of undetermined significance (ASCUS) or worse, referred directly to colposcopy biopsy; 2) cytology normal and HPV16/18 positive, referred directly to colposcopy biopsy $[4,7]$.

Between December 2012 and April 2015, a total of 19207 consecutive women (median age 41.3 years; range 16-89) underwent cervical cancer screening in gynecological clinic at Taizhou Hospital of Zhejiang Province. The flowchart of study population was shown on Figure 1. Our final sample of 1648 women underwent colposcopy biopsy within 12 weeks. The study excluded hysterectomy, a history of cervical cancer, no treatment for CIN in the preceding 12 months or infection with HIV. Informed consent was obtained from participants in the study. For those participants younger than 18 years old, the consent form was signed by the parents of each participant.

\section{HPV genotyping}

HPV genotyping was performed using the GP5+/ bioGP6+-PCR/MPG assay, which was approved by the China Food and Drug Administration (CFDA Certified NO. (2014): 3400847). DNA was extracted from $200 \mu \mathrm{l}$ of each sample and collected in $50 \mu$ l elution buffer according to the manufacturer's instructions. $5 \mu l$ of extracted DNA was amplified by the GP5+/bioGP6+ broad-spectrum primer set with a final volume of $20 \mu l$. Reactions were heated for $5 \mathrm{~min}$ at $95^{\circ} \mathrm{C}$, followed by 35 repeated cycles of $94^{\circ} \mathrm{C}$ for $30 \mathrm{~s}, 55^{\circ} \mathrm{C}$ for $30 \mathrm{~s}, 72^{\circ} \mathrm{C}$ for $30 \mathrm{~s}$, and a final extension step at $72^{\circ} \mathrm{C}$ for $10 \mathrm{~min}$. Following the PCR amplification, $3 \mu 1$ of biotin-labeled PCR products and $22 \mu$ l of hybridization solution containing 27 types of coupled beads of each set were transferred to 96-well plates. Hybridization was performed at $95^{\circ} \mathrm{C}$ for $5 \mathrm{~min}$ followed by $48^{\circ} \mathrm{C}$ for $30 \mathrm{~min}$. Subsequently, streptavidinphycoerythrin was added to each well at $48^{\circ} \mathrm{C}$ for $15 \mathrm{~min}$. The biotin-labeled PCR products were captured by HPV type-specific probes attached to color-coded beads, streptavidin-phycoerythrin was used as the reporter bound to the target, and the HPV genotypes were analyzed using the Luminex $200^{\mathrm{TM}}$ analyzer.

In short, it comprises the GP5+/bioGP6+-PCR, which using sets of biotinylated amplimers and a multiplex human papillomavirus genotyping (MPG) methods with bead-based Luminex suspension array technology $[8,9]$, which is able to simultaneously identify $14 \mathrm{hrHPV}$ types including $16,18,31,33,35,39,45,51,52,56,58,59,66$, 68 and 12 low-risk HPV (lrHPV) types including 6, 11, $26,40,42,44,53,55,61,73,82,83$ and $\beta$-globin gene (internal control).

\section{Diagnostic procedure}

Cytological results, which blinded to the outcomes of HPV testing, were reported according to the 2001 Bethesda system. Cytological results were grouped as negative for intraepithelial lesion or malignancy (NILM), ASCUS, atypical squamous cells and cannot exclude highgrade squamous intraepithelial lesions (ASC-H), lowgrade squamous intraepithelial lesions (LSIL), high-grade squamous intraepithelial lesions (HSIL), squamous cell carcinoma, atypical glandular cells (AGC), endocervical adenocarcinoma in situ (AIS), and adenocarcinoma.

Histopathologic diagnoses were adjudicated by pathologists and classified as normal, CIN grade 1, 2, 3 or invasive cervical cancer, according to international criteria. The suffix "+" means the indicated histology or more severe. Disease end points were histopathologically confirmed CIN2+ or CIN3+.

\section{Statistical analysis}

Performance characteristics of hrHPV test (sensitivity, specificity, positive predictive value, negative predictive value) for identification of CIN2+ (to include CIN2, CIN3, adenocarcinoma in situ, and ICC) were determined using standard statistical tests. The chisquared and Fisher's exact tests were used to evaluate relative CIN2+ risk associate with HPV genotypes, odds ratios (ORs) and relative 95\% confidence interval (CI). All 
Table 1: Clinical characteristics of the study population $(n=1648)$

\begin{tabular}{|l|l|l|l|l|l|}
\hline Characteristic & $\begin{array}{l}\text { Cervicitis } \\
(\boldsymbol{n}=\mathbf{1 0 8 1})\end{array}$ & $\begin{array}{l}\text { CIN1 } \\
(\boldsymbol{n}=\mathbf{2 2 6})\end{array}$ & $\begin{array}{l}\text { CIN2 } \\
(\boldsymbol{n}=\mathbf{1 2 2})\end{array}$ & $\begin{array}{l}\text { CIN3 } \\
(\boldsymbol{n}=\mathbf{1 8 6})\end{array}$ & $\begin{array}{l}\text { Cervical cancer } \\
(\boldsymbol{n}=\mathbf{3 3})\end{array}$ \\
\hline Age & $\mathbf{4 2 . 1} \pm \mathbf{1 0 . 5}$ & $\mathbf{4 0 . 6} \pm \mathbf{1 1 . 0}$ & $\mathbf{4 1 . 4} \pm \mathbf{9 . 1}$ & $\mathbf{4 3 . 9} \pm \mathbf{8 . 9}$ & $\mathbf{5 1 . 3} \pm \mathbf{9 . 9 *}$ \\
\hline Primary hrHPV Genotyping Strategy & 885 & 195 & 117 & 169 & 33 \\
\hline Current Cervical Screening Strategy & 309 & 95 & 60 & 81 & 22 \\
\hline
\end{tabular}

Abbreviations: CIN, cervical intraepithelial neoplasia; hrHPV, high-risk human papillomavirus.

$*, P<0.05$ vs. each other groups.

Table 2: Prevalence rates of hrHPV genotypes for cervical pathology status among hrHPV-positive women $(n=1399)$

\begin{tabular}{|c|c|c|c|c|c|c|c|c|c|c|}
\hline \multirow{2}{*}{$\begin{array}{l}\text { hrHPV } \\
\text { genotypes }\end{array}$} & \multicolumn{2}{|c|}{ Cervical cancer $(n=33)$} & \multicolumn{2}{|c|}{$\begin{array}{l}\text { CIN3 } \\
(n=169) \\
\end{array}$} & \multicolumn{2}{|c|}{\begin{tabular}{|l|} 
CIN2 \\
$(n=117)$ \\
\end{tabular}} & \multicolumn{2}{|c|}{\begin{tabular}{|l|} 
CIN1 \\
$(n=195)$ \\
\end{tabular}} & \multirow[b]{2}{*}{$n$} & \multirow{2}{*}{$\begin{array}{l}\begin{array}{l}\text { Cervicitis } \\
(n=885)\end{array} \\
\%(95 \% C I)\end{array}$} \\
\hline & $n$ & $\%(95 \% \mathrm{CI})$ & $n$ & $\%(95 \% \mathrm{CI})$ & $n$ & $\%(95 \% \mathrm{CI})$ & $n$ & $\%(95 \% \mathrm{CI})$ & & \\
\hline HPV 52 & 4 & $12.1(1.0-23.3)$ & 27 & $16.0(10.5-21.5)$ & 30 & $25.6(17.7-33.6)$ & 57 & $29.2(22.8-35.6)$ & 235 & $26.6(23.6-29.5)$ \\
\hline HPV 16 & 20 & $60.6(43.9-77.3)$ & 81 & $47.9(40.4-55.5)$ & 37 & $31.6(23.2-40.0)$ & 33 & $16.9(11.7-22.2)$ & 123 & $13.9(11.6-16.2)$ \\
\hline HPV 58 & 3 & $9.1(0.0-18.9)$ & 34 & $20.1(14.1-26.2)$ & 25 & $21.4(13.9-28.8)$ & 28 & $14.4(9.4-19.3)$ & 139 & $15.7(13.3-18.1)$ \\
\hline HPV 39 & 0 & $0.0(0.0-0.0)$ & 6 & $3.6(0.8-6.3)$ & 6 & $5.1(1.1-9.1)$ & 14 & 7.2(3.6-10.8) & 88 & $9.9(8.0-11.9)$ \\
\hline HPV 56 & 0 & $0.0(0.0-0.0)$ & 3 & $1.8(0.0-3.8)$ & 9 & $7.7(2.9-12.5)$ & 14 & 7.2(3.6-10.8) & 83 & $9.4(7.5-11.3)$ \\
\hline HPV 18 & 3 & $9.1(0.0-18.9)$ & 9 & $5.3(1.9-8.7)$ & 6 & 5.1(1.1-9.1) & 16 & $8.2(4.4-12.1)$ & 75 & $8.5(6.6-10.3)$ \\
\hline HPV 68 & 1 & $3.0(0.0-8.9)$ & 2 & $1.2(0.0-2.8)$ & 2 & $1.7(0.0-4.1)$ & 9 & $4.6(1.7-7.6)$ & 77 & 8.7(6.8-10.6) \\
\hline HPV 33 & 2 & $6.1(0.0-14.2)$ & 25 & $14.8(9.4-20.1)$ & 16 & 13.7(7.4-19.9) & 14 & 7.2(3.6-10.8) & 57 & $6.4(4.8-8.1)$ \\
\hline HPV 59 & 0 & $0.0(0.0-0.0)$ & 9 & $5.3(1.9-8.7)$ & 5 & 4.3(0.6-7.9) & 19 & $9.7(5.6-13.9)$ & 55 & 6.2(4.6-7.8) \\
\hline HPV 51 & 0 & $0.0(0.0-0.0)$ & 7 & 4.1(1.1-7.1) & 5 & 4.3(0.6-7.9) & 11 & $5.6(2.4-8.9)$ & 44 & $5.0(3.5-6.4)$ \\
\hline HPV 31 & 3 & 9.1(0.0-18.9) & 8 & $4.7(1.5-7.9)$ & 13 & 11.1(5.4-16.8) & 13 & $6.7(3.2-10.2)$ & 35 & $4.0(2.7-5.2)$ \\
\hline HPV 66 & 0 & $0.0(0.0-0.0)$ & 0 & $0.0(0.0-0.0)$ & 2 & $1.7(0.0-4.1)$ & 9 & $4.6(1.7-7.6)$ & 38 & 4.3(3.0-5.6) \\
\hline HPV 35 & 0 & $0.0(0.0-0.0)$ & 2 & $1.2(0.0-2.8)$ & 0 & $0.0(0.0-0.0)$ & 2 & $1.0(0.0-2.4)$ & 16 & $1.8(0.9-2.7)$ \\
\hline HPV 45 & 0 & $0.0(0.0-0.0)$ & 7 & $4.1(1.1-7.1)$ & 1 & $0.9(0.0-2.5)$ & 2 & $1.0(0.0-2.4)$ & 15 & $1.7(0.8-2.5)$ \\
\hline
\end{tabular}

Abbreviations: CIN, cervical intraepithelial neoplasia; hrHPV, high-risk human papillomavirus.

* Women with multiple HPV types detected are counted to each type, and therefore counted more than once.

statistical analyses were performed using SPSS version 15.0 (SPSS Inc., Chicago, IL). $P$ values were two-sided, and statistical significance was accepted if the $P$ value was 0.05 or less.

\section{RESULTS}

\section{Characteristics of the population}

As shown in Table 1 and Figure 1, our final sample of 1648 women had diagnosed with biopsy, including 1081 women who diagnosed with cervicitis, 226 with CIN1, 122 with CIN2, 186 with CIN3, and 33 with ICC, respectively. The average ages of women with cervicitis, CIN1, CIN2, CIN3, and ICC were 42.1 $\pm 10.5,40.6 \pm 11.0$, $41.4 \pm 9.1,43.9 \pm 8.9$, and 51.3 \pm 9.9 , respectively. Women with cervical cancer were significantly older than those in the other groups $(P<0.001)$. According our recommended hrHPV genotyping for primary cervical screening strategy,
1399 women underwent colposcopy biopsy. According the current cervical screening strategy, 567 women underwent colposcopy biopsy.

\section{Prevalence of HPV genotypes}

The overall prevalence of HPV was $22.2 \%(95 \%$ CI 21.6-22.8\%), hrHPV and lrHPV infection rates were $18.2 \%$ (95\% CI 17.6-18.7\%) and 4.0\% (95\% CI 3.7$4.3 \%$ ), respectively. Overall, HPV52 was the most prevalent genotype $(4.9 \%)$, either alone or in combination with other types, followed by 16 (3.1\%), 58 (2.7\%), 39 (1.6\%), 18 (1.5\%), 56 (1.5\%) (Table S1).

To assess the clinical predictive value for different hrHPV types, we further evaluated our data on the prevalence of individual hrHPV infection rates for cervical pathology status (Table2 and Figure2). For the patients with cervicitis/CIN1, HPV52 was the most common HPV type with the prevalence of $27.0 \%$, followed by 58 (15.5\%), $16(14.4 \%), 39(9.4 \%), 56(9.0 \%)$ and 18 


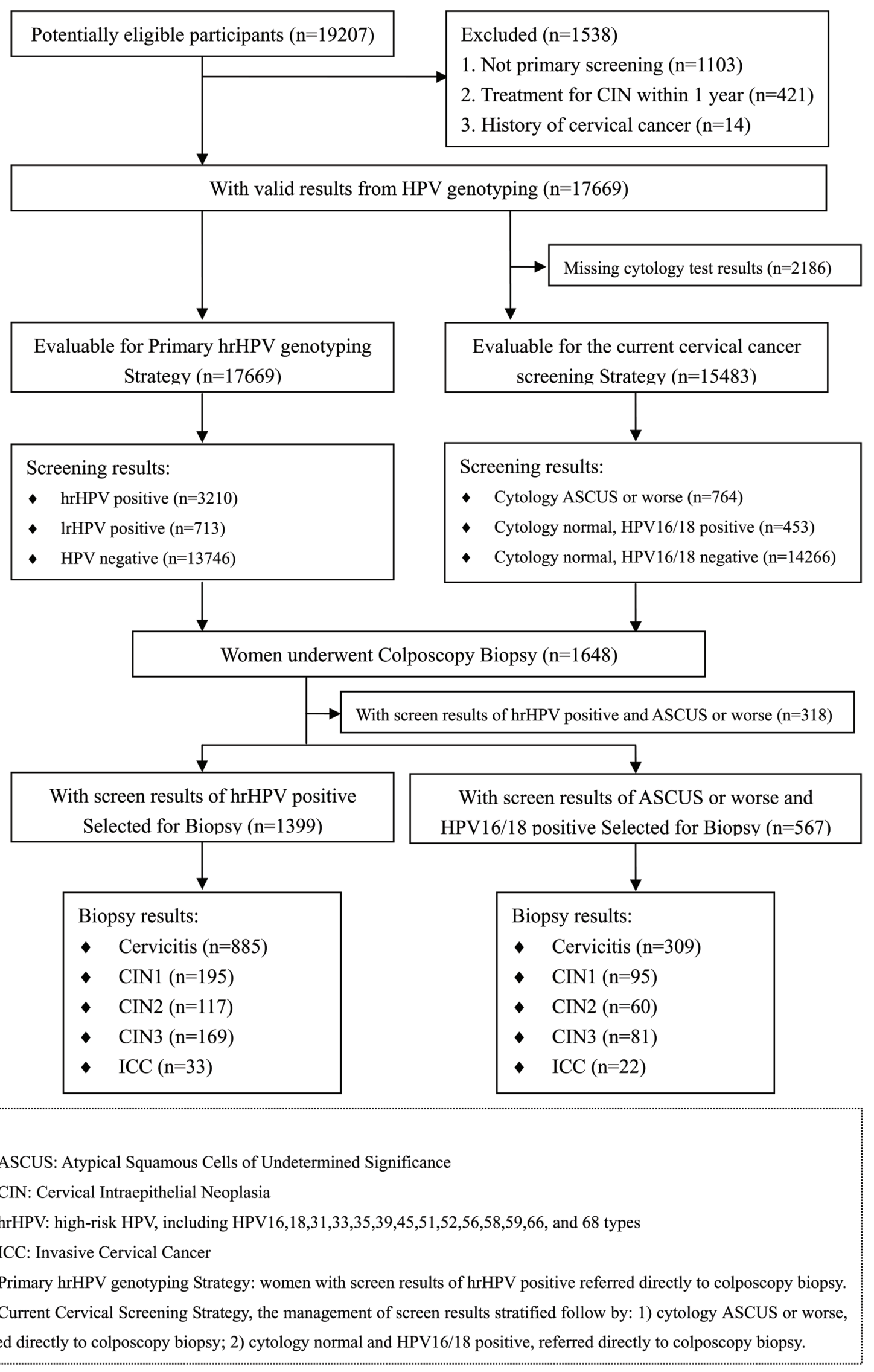

Figure 1: Flow chart of inclusion and exclusion criteria of the study population. 
Table3: The accuracy values of different triage strategies for the detection of CIN2+/CIN3+

\begin{tabular}{|c|l|l|l|}
\hline Screen Strategy & $\begin{array}{l}\text { Performance } \\
\text { measure } \\
(\mathbf{9 5 \%} \mathbf{C I})\end{array}$ & CIN2+ & CIN3+ \\
\hline $\begin{array}{l}\text { Current cervical } \\
\text { cancer screening }\end{array}$ & Sensitivity & $71.1(65.2-77.0)$ & $71.4(63.9-78.9)$ \\
\hline & Specificity predictive & $28.4(24.6-32.1)$ & $60.0(57.2-62.8)$ \\
\hline $\begin{array}{l}\text { Positive } \\
\text { value } \\
\text { value } \\
\text { genotyping test }\end{array}$ & $\begin{array}{l}\text { Negative predictive } \\
\text { Sensitivity }\end{array}$ & $91.2(89.1-93.2)$ & $17.7(14.6-20.9)$ \\
\hline & Specificity & $17.5(15.3-19.4)$ & $94.6(92.9-96.2)$ \\
\hline & $\begin{array}{l}\text { Positive predictive } \\
\text { value }\end{array}$ & $22.8(20.6-25.0)$ & $92.2(88.7-95.8)$ \\
\hline $\begin{array}{l}\text { Negative predictive } \\
\text { value }\end{array}$ & $91.2(87.6-94.7)$ & $17.8(15.7-19.8)$ \\
\hline
\end{tabular}

Abbreviations: CIN, cervical intraepithelial neoplasia; hrHPV, high-risk human papillomavirus; CI: 95\% confidence interval.

(8.4\%). For the patients with CIN2+, HPV16 was the most common HPV type with the prevalence of $43.3 \%$, followed by $58(19.4 \%), 52(19.1 \%), 33(13.5 \%), 31(7.5 \%)$ and $18(5.6 \%)$. HPV16, 52 and 58 were the three HPV types most commonly found in any cervical pathology status. Notably, HPV52 was the most common type among women with cervicitis/CIN1, but the distribution changed remarkably for $\mathrm{CIN} 2+$, where ranked the third.

\section{Primary hrHPV genotyping strategy}

In this study, we recommended hrHPV genotyping for primary cervical screening strategy, when women with screen results of hrHPV positive referred directly

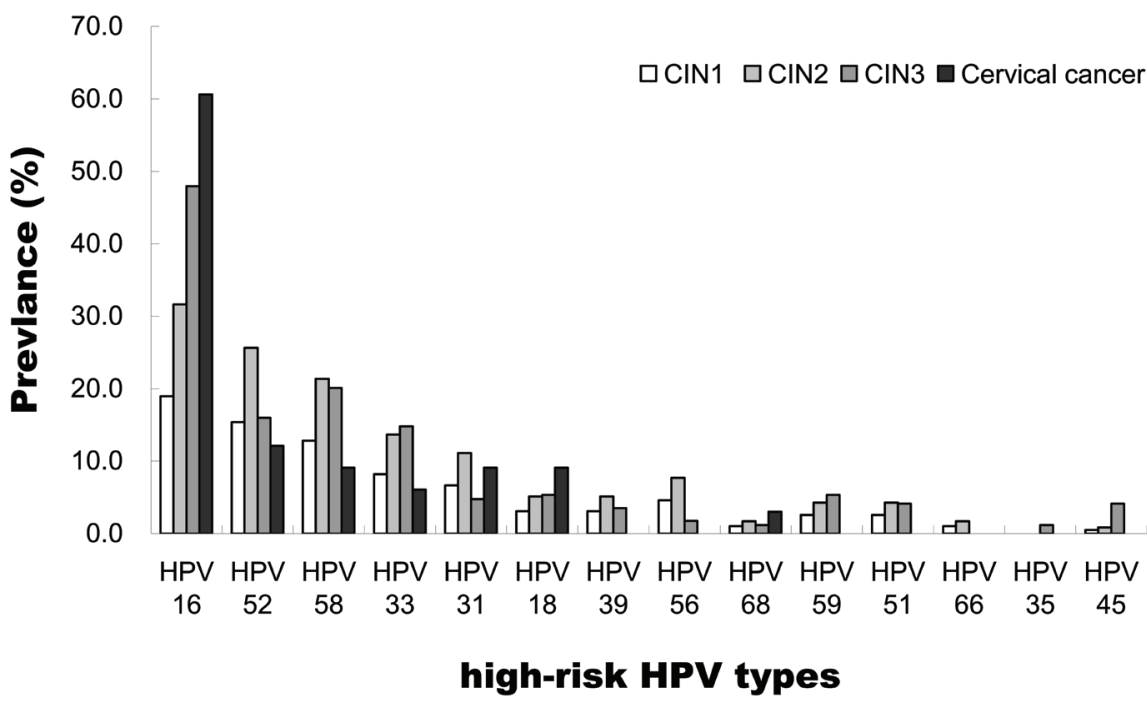

Figure 2: Relative distribution of high-risk HPV genotypes among HPV-positive cervical intraepithelial neoplasia (CIN; graded from 1 to 3) and cervical cancer women. Women with multiple HPV types detected are counted to each type, and therefore counted more than once. to biopsy. According to this guideline, 3210 women need biopsy, irrespective of women age. In fact, 1399 (43.6\%) women with hrHPV infection accepted biopsy, including 885 women with cervicitis, 195 with CIN1, 117 with CIN2, 169 with CIN3, and 33 with ICC, respectively. According this screening strategy, the sensitivity and specificity for detecting CIN2+ were $93.5 \%$ and $17.5 \%$, respectively. The positive predictive value and negative predictive value were $22.8 \%$ and $91.2 \%$, respectively.

According to the current cervical screening strategy, 1217 women need biopsy, irrespective of women age. In fact, 567 (46.6\%) women with ASCUS+ or HPV16/18 positive accepted biopsy, including 309 women with cervicitis, 95 with CIN1, 60 with CIN2, 81 with CIN3, and

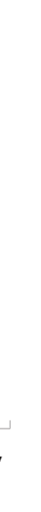

$\square$ CIN1 $\square$ CIN2 $\square$ CIN3 $\square$ Cervical cancer 
22 with ICC, respectively. The sensitivity and specificity for detecting CIN2+ were $71.1 \%$ and $62.4 \%$, respectively. The positive predictive value and negative predictive value were $28.4 \%$ and $91.1 \%$, respectively. Compared to the current cervical screening, primary hrHPV genotyping test had higher sensitivity and lower specificity (Table 3).

\section{Management of women with hrHPV infection}

We further examined whether hrHPV positive women for HPV16/18 (2 types), or HPV16/18/31/33/52/58 (the 6 major carcinogenic types) can serve as a triage tool to discriminate women who need biopsy immediately. Compared to 12 other hrHPV infection women, the ORs for CIN2+ in HPV16/18 (2 types) infection women was 2.7 (95\%CI 2.1-3.4). Notably, the relative CIN2+ risk (ORs) was 3.2(95\%CI 2.3-4.1) for women with HPV16/18/31/33/52/58 (6 types) infection compared to women with HPV35/39/45/51/56/59/66/68 infection. For detecting CIN2+, the sensitivity and specificity were $86.5 \%$ and $43.5 \%$, respectively. The positive predictive value and negative predictive value were $28.5 \%$ and $92.5 \%$, respectively. Therefore, when women with HPV16/18/31/33/52/58 infection can be recommended colposcopy biopsy immediately.

Women who are HPV35/39/45/51/56/59/66/68 positive should be detected with cervical cytological testing. Among women with ASCUS cytology, HPV35/39/45/51/56/59/66/68 infection rate was $32.4 \%$. Women with ASCUS+ can be recommended colposcopy biopsy immediately. The accuracy values of different triage strategies for the detection of CIN2+/CIN3+ were shown in Table S2.

\section{DISCUSSION}

In recent years, hrHPV testing for triaging ASCUS and co-testing with cervical cytology have been implemented in clinical practice $[4,10]$. However, clinical data for primary HPV screening alone are currently lacking [5, 11]. Numerous of prospective randomized screening trials, primarily from Europe or United States, have documented that co-testing offers minimal increased protection against the subsequent progression of cervical lesions compared to primary HPV testing, which is more sensitive than cytology screening, but specificity depends on subsequent evaluation strategies and screening frequencies [10-13]. Recently, American FDA approval HPV testing as an option for primary screening [5], however, clinical practice guidelines for primary HPV screening strategy, which do not exist in China. It is important to improve knowledge about the complete carcinogenic process for individual hrHPV genotypes from infection to cervical cancer, which may serve as predictive markers of disease persistence and progression. Therefore, our study addressed an important question is to detect hrHPV genotypes initial cytology specimens of whether associated with the progression of high-grade cervical lesions during pathology diagnose, in order to reduce the number of biopsy and improve the CIN2+ detection rate, further renewed the cervical cancer screening strategies in China.

The prevalence of hrHPV (18.2\%) obtained in this present study were similar to that in Hangzhou (19.9\%) and Nanchang (18.4\%) which also region in southeast of China [14]. Consistent with the data generated by Chinese population-based investigations, HPV16, HPV52, and HPV58 were found to be the dominant hrHPV types [14, 15], but unlike in a meta-analysis that summarized global reports [16] in which HPV16, 18, and 45, HPV16, 18, and 33 or HPV16, 18, and 58 were most commonly detected. In our population, HPV52 and HPV58 accounted for $26.4 \%$, which are all common among Asian populations and markedly higher than the global rate of $14.0 \%$ [16]. HPV52 was detected in $26.6 \%, 29.2 \%$, and $19.1 \%$ of women with cervicitis, CIN1, and CIN2+, respectively. HPV 58 was detected in $15.7 \%, 14.4 \%$, and $19.4 \%$ of women with cervicitis, CIN1, and CIN2+, respectively. These data showed that HPV52 is more common among cervicitis women, whereas HPV58 is more common among CIN2+ women, which was also confirmed by several other studies [16-19]. HPV58, which is associated with a higher risk of developing high-grade cervical lesions than other non-HPV16 types [20], has been found in a relatively higher proportion of women with highgrade cervical lesions in Eastern Asia than elsewhere [16, 21].

In our population, we found that HPV16, 31, 33, and 58 increased the risk for CIN1 lesions progress to CIN2 or worse (Figure2). HPV genotyping test will enable us to characterize a woman's cervical disease risk more precisely, the OR for CIN2+ in HPV16/18/31/33/52/58 positive women was 3.2 (95\%CI $2.3-4.1)$ when compared to HPV35/39/45/51/56/59/66/68 positive women. Consistent with the data generated by global meta-analysis, HPV16/18/31/33/52/58 are the six most common genotypes detected in women with cervical cancer worldwide, according for $>90 \%$ of cervical cancer in each area [16, 22-24]. In 2015, the Advisory Committee on Immunization Practices (ACIP) recommended 9-valent HPV vaccine (9vHPV) which contains HPV6, 11, 16, $18,31,33,45,52$, and 58 virus-like particle (VLP) was licensed by the FDA $[25,26]$. The $9 v H P V$ vaccine covers the 6 major carcinogenic HPV genotypes which prevalent approximately $70 \%$ of hrHPV infection in Taizhou area. These findings defined principles for the national population-based screening programs and vaccination in southeast China.

Compared with current cervical cancer screening strategy, we recommended screening strategy had higher sensitivity $(93.5 \%)$ and higher negative predictive 
values $(91.2 \%)$. In the present study, the current cervical screening strategy had been missing the majority of women (178 cases, 52.2\%) with hrHPV infections who progress to high-grade cervical lesions (CIN2+) (Table1). Our results supported that HPV16/18/31/33/52/58-positive women need immediate biopsy, which would increase the number of CIN2+ by approximately doubling. In a 14year follow-up of a randomized primary HPV screening, HPV16/18/31/33/45/52/58 had 14-year cumulative incidences $73.9 \%$ of CIN2+ and all hrHPV genotypes contributed $86.9 \%$ [27], and $30 \%$ of cervical cancers are associated with hrHPV genotypes other than HPV16 and HPV18 [28]. In addition, it has been reported that the reassurance of HPV-negative women with primary HPV screening every 3 years was nearly equivalent to cotesting every 5 years [12]. The 18-year follow-up analysis showed that hrHPV-positive women were more likely to be diagnosed with CIN2+ $(P<0.001) 10-18$ years after enrollment compared with hrHPV-negative women. The 18-year cumulative incidence rates (CIRs) of CIN2+ among hrHPV-positive and hrHPV-negative women were $23.2 \%$ and $1.5 \%$, respectively. [29]. These findings support the hrHPV genotyping for primary cervical screening strategy could replace co-testing.

However, poor specificity (17.5\%) and poor positive predictive value $(22.8 \%)$ for the determination of CIN2+ in the current study would be limits the use of our recommended screening strategy. In our study, women with HPV16/18/31/33/52/58 (the 6 major carcinogenic types) infections rate were $71.1 \%$ (2282/3210) of overall hrHPV-positive women. In order to reduce the number of biopsy, we suggested when women with HPV16/18/31/33/52/58 infection can be recommended colposcopy biopsy immediately. For detecting CIN2+, the specificity and positive predictive value were $43.5 \%$ and $28.5 \%$, respectively. In order to improve the CIN2+ detection rate, our data suggested that reflex cytology for women with HPV35/39/45/51/56/59/66/68 infection will be clinically useful as a triage test tool for immediate biopsy for women with ASCUS or worse.

In summary, hrHPV genotyping provide a better predictive value than HPV16/18 genotyping alone in guiding the clinical management of the current cervical cancer screening. HPV testing without adjunctive cytology may be sufficiently sensitive for primary cervical cancer screening.

\section{ACKNOWLEDGMENTS}

This work was supported by the grants from National Natural Science Foundation of China (31370920, 81372247), Science and Technology Bureau of Zhejiang Province (2016C33231), Health Bureau of Zhejiang Province (2015KYB438) and Zhejiang Provincial program for the cultivation of high-level innovative health talents.

\section{CONFLICTS OF INTERESTS}

The authors declare that they have no competing interests.

\section{Author Contributions}

Designed the experiments: WHY HHX. HPV genotyping: HHX WWS. Cytology testing and pathological diagnosis: AFL XJS YZ. Performed statistical analysis and drafted the manuscript: HHX WHY WWS. All authors read and approved the final manuscript.

\section{REFERENCES}

1. Crosbie EJ, Einstein MH, Franceschi S, Kitchener HC. Human papillomavirus and cervical cancer. Lancet. 2013; 382: 889-99.

2. de Sanjose S, Quint WG, Alemany L, Geraets DT, Klaustermeier JE, Lloveras B, Tous S, Felix A, Bravo LE, Shin HR, Vallejos CS, de Ruiz PA, Lima MA, et al. Human papillomavirus genotype attribution in invasive cervical cancer: a retrospective cross- sectional worldwide study. Lancet Oncol. 2010; 11:1048-56.

3. Smith RA, Brooks D, Cokkinides V, Saslow D, Brawley OW. Cancer screening in the United States, 2013: a review of current American Cancer Society guidelines, currentissues in cancer screening, and new guidance on cervical cancer screening and lung cancer screening. CA Cancer J Clin. 2013; 63: 88-105.

4. Saslow D, Solomon D, Lawson HW, Killackey M, Kulasingam SL, Cain J, Garcia FA, Moriarty AT, Waxman AG, Wilbur DC, Wentzensen N, Downs LS Jr, Spitzer $\mathrm{M}$, et al. American Cancer Society, American Society for Colposcopy and Cervical Pathology, and American Society for Clinical Pathology screening guidelines for the prevention and early detection of cervical cancer. CA Cancer J Clin. 2012; 62:147-72.

5. Huh WK, Ault KA, Chelmow D, Davey DD, Goulart RA, Garcia FA, Kinney WK, Massad LS, Mayeaux EJ, Saslow D, Schiffman M, Wentzensen N, Lawson HW, et al. Use of primary high-risk human papillomavirus testing for cervical cancer screening: interim clinical guidance. Gynecol Oncol. 2015; 136:178-82.

6. Lyons YA, Kamat AA, Zhou H, Mody DR, Schwartz MR, Hobday C, Ge Y. Non-16/18 high-risk HPV infection predicts disease persistence and progression in women with an initial interpretation of LSIL. Cancer Cytopathol. 2015; 123:435-42.

7. Wright TC Jr, Massad LS, Dunton CJ, Spitzer M, Wilkinson EJ, Solomon D; 2006 American Society for Colposcopy and Cervical Pathology-sponsored Consensus Conference. 2006 consensus guidelines for the management of women with 
abnormal cervical screening tests. Am J Obstet Gynecol. 2007; 197: 346-55.

8. Jiang HL, Zhu HH, Zhou LF, Chen F, Chen Z. Genotyping of human papillomavirus in cervical lesions by L1 consensus PCR and the Luminex xMAP system. J Med Microbiol. 2006; 55:715-20.

9. Schmitt M, Depuydt C, Benoy I, Bogers J, Antoine J, Pawlita M, Arbyn M; VALGENT study group. Viral load of high-risk human papillomaviruses as reliable clinical predictor for the presence of cervical lesions. Cancer Epidemiol Biomarkers Prev. 2013; 22:406-14.

10. Stoler MH, Wright TC Jr, Sharma A, Apple R, Gutekunst $\mathrm{K}$, Wright TL, ATHENA (Addressing THE Need for Advanced HPV Diagnostics) HPV Study Group. Highrisk human papillomavirus testing in women with ASC-US cytology: results from the ATHENA HPV study. Am J Clin Pathol. 2011; 135:468-75.

11. Ronco G, Dillner J, Elfström KM, Tunesi S, Snijders PJ, Arbyn M, Kitchener H, Segnan N, Gilham C, GiorgiRossi P, Berkhof J, Peto J, Meijer CJ; International HPV screening working group. Efficacy of HPV-based screening for prevention of invasive cervical cancer: follow-up of four European randomized controlled trials. Lancet. 2014; 383: 524-32.

12. Gage JC, Schiffman M, Katki HA, Castle PE, Fetterman B, Wentzensen N, Poitras NE, Lorey T, Cheung LC, Kinney WK. Reassurance against future risk of precancer and cancer conferred by a negative human papillomavirus test. J Natl Cancer Inst. 2014; 106. pii: dju153.

13. Katki HA, Kinney WK, Fetterman B, Lorey T, Poitras NE, Cheung L, Demuth F, Schiffman M, Wacholder S, Castle PE. Cervical cancer risk for women undergoing concurrent testing for human papillomavirus and cervical cytology: a population-based study in routine clinical practice. Lancet Oncol. 2011; 12:663-72.

14. Wang R, Guo XL, Wisman GB, Schuuring E, Wang WF, Zeng ZY, Zhu H, Wu SW. Nationwide prevalence of human papillomavirus infection and viral genotype distribution in 37 cities in China. BMC Infect Dis. 2015; 15:257.

15. Li J, Huang R, Schmidt JE, Qiao YL. Epidemiological features of Human Papillomavirus (HPV) infection among women living in Mainland China. Asian Pac J Cancer Prev. 2013; 14:4015-23.

16. Guan P, Howell-Jones R, Li N, Bruni L, de Sanjosé S, Franceschi S, Clifford GM. Human papillomavirus types in 115,789 HPV-positive women: a meta-analysis from cervical infection to cancer. Int J Cancer. 2012; 131:234959.

17. Jing L, Zhong X, Huang W, Liu Y, Wang M, Miao Z, Zhang X, Zou J, Zheng B, Chen C, Liang X, Yang G, Jing $\mathrm{C}$, et al. HPV genotypes and associated cervical cytological abnormalities in women from the Pearl River Delta region of Guangdong province, China: a cross-sectional study. BMC Infect Dis. 2014; 14:388.
18. Li J, Mei J, Wang X, Hu L, Lin Y, Yang P. Human papillomavirus type-specific prevalence in women with cervical intraepithelial neoplasm in Western China. J Clin Microbiol. 2012; 50:1079-81.

19. Chan PK, Cheung TH, Li WH, Yu MY, Chan MY, Yim SF, Ho WC, Yeung AC, Ho KM, Ng HK. Attribution of human papillomavirus types to cervical intraepithelial neoplasia and invasive cancers in Southern China. Int J Cancer. 2012; 131:692-705.

20. Chen HC, Schiffman M, Lin CY, Pan MH, You SL, Chuang LC, Hsieh CY, Liaw KL, Hsing AW, Chen CJ; CBCSPHPV Study Group. Persistence of type-specific human papillomavirus infection and increased long-term risk of cervical cancer. J Natl Cancer Inst. 2011; 103:1387-96.

21. Gu Y, Ma C, Zou J, Zhu Y, Yang R, Xu Y, Zhang Y. Prevalence characteristics of high-risk human papillomaviruses in women living in Shanghai with cervical precancerous lesions and cancer. Oncotarget. 2016; 7:24656-63. doi: 10.18632/oncotarget.8262.

22. Smith JS, Lindsay L, Hoots B, Keys J, Franceschi S, Winer R, Clifford GM. Human papillomavirus type distribution in invasive cervical cancer and high-grade cervical lesions: a meta-analysis update. Int J Cancer. 2007; 121:621-32.

23. Tjalma WA, Fiander A, Reich O, Powell N, Nowakowski AM, Kirschner B, Koiss R, O'Leary J, Joura EA, Rosenlund M, Colau B, Schledermann D, Kukk K, et al. Differences in human papillomavirus type distribution in high-grade cervical intraepithelial neoplasia and invasive cervical cancer in Europe. Int J Cancer. 2013; 132: 854-67.

24. Ding DC, Hsu HC, Huang RL, Lai HC, Lin CY, Yu MH, Chu TY. Type-specific distribution of HPV along the full spectrum of cervical carcinogenesis in Taiwan: an indication of viral oncogenic potential. Eur J Obstet Gynecol Reprod Biol. 2008; 140:245-51.

25. Petrosky E, Bocchini JA Jr, Hariri S, Chesson H, Curtis CR, Saraiya M, Unger ER, Markowitz LE; Centers for Disease Control and Prevention (CDC). Use of 9-valent human papillomavirus (HPV) vaccine: updated HPV vaccination recommendations of the advisory committee on immunization practices. MMWR Morb Mortal Wkly Rep. 2015; 64:300-4.

26. Food and Drug Administration. December 10, 2014 Approval letter-GARDASIL 9. Silver Spring, MD: US Department of Health and Human Services, Food and Drug Administration; 2014. Available at http://www.fda.gov/ BiologicsBloodVaccines/Vaccines/ApprovedProducts/ ucm426520.htm.

27. Smelov V, Elfström KM, Johansson AL, Eklund C, Naucler P, Arnheim-Dahlström L, Dillner J. Long-term HPV typespecific risks of high-grade cervical intraepithelial lesions: a 14-year follow-up of a randomized primary HPV screening trial. Int J Cancer. 2015; 136:1171-80.

28. Massad LS, Einstein MH, Huh WK, Katki HA, Kinney WK, Schiffman M, Solomon D, Wentzensen N, Lawson HW; 2012 ASCCP Consensus Guidelines Conference. 
2012 updated consensus guidelines for the management of abnormal cervical cancer screening tests and cancer precursors. Obstet Gynecol. 2013; 121:829-46.

29. Castle PE, Glass AG, Rush BB, Scott DR, Wentzensen N, Gage JC, Buckland J, Rydzak G, Lorincz AT, Wacholder S. Clinical human papillomavirus detection forecasts cervical cancer risk in women over 18 years of follow-up. J Clin Oncol. 2012; 30:3044-50. 